\title{
HALP Score as a New Prognostic Index in Metastatic Renal Cell Cancer
}

\author{
Ferhat Ekinci ${ }^{1}$, Onur Yazdan Balcik ${ }^{2}$, Esin Oktay ${ }^{2}$ and Atike Pinar Erdogan ${ }^{1}$ \\ ${ }^{1}$ Department of Medical Oncology, Celal Bayar University, Manisa, Turkey \\ ${ }^{2}$ Department of Medical Oncology, Adnan Menderes University, Aydin, Turkey
}

\begin{abstract}
Objective: To evaluate prognostic significance of the new index, designed by formulating hemoglobin, albumin, lymphocyte, and platelet (HALP) counts in patients with metastatic renal cell carcinoma (RCC).

Study Design: Descriptive study.

Place and Duration of Study: Department of Medical Oncology, Celal Bayar University, Manisa, Turkey and Adnan Menderes University, Aydin, Turkey, from January 2014 to April 2020.

Methodology: Patients with metastatic RCC and sufficient follow-up data were included in the study as a retrospective cohort. HALP score was calculated as hemoglobin $(\mathrm{g} / \mathrm{L}) \times$ albumin $(\mathrm{g} / \mathrm{L})$ levels $\times$ lymphocyte count $(/ \mathrm{L}) /$ platelet count $(/ \mathrm{L})$. The cut-off value was determined by examining the area under the ROC curve for the HALP value. The endpoints of this study included overall survival (OS) and progression-free survival (PFS).

Results: The mean overall survival (OS) of the patients with low HALP score was 17.7 months (95\% Cl, $2.21-33.18)$, while the OS of the patients with high HALP score was 89.7 months $(95 \% \mathrm{Cl}, 55.62-123.77)$ and reached statistical significance $(p=0.001)$. The results of univariate $(p=0.009)$ and multivariate $(p=0.012)$ analyses were statistically significant as well.

Conclusion: The HALP score in metastatic RCC patients was closely related to the prognosis. Worse OS was found in patients with a low HALP score.
\end{abstract}

Key Words: HALP score, Overall survival, Progression-free survival, Renal cell carcinoma.

How to cite this article: Ekinci F, Balcik OY, Oktay E, Erdogan AP. HALP Score as a New Prognostic Index in Metastatic Renal Cell Cancer. J Coll Physicians Surg Pak 2022; 32(03):313-318.

\section{INTRODUCTION}

Renal cell carcinoma (RCC) is the most common type of kidney cancer and constitutes $2 \%$ to $5 \%$ of all cancers with an increasing incidence. ${ }^{1,2}$ At the time of diagnosis, it may be metastatic at a rate of almost $20 \%$ and the risk of recurrence may be $40 \%$, even ifitis detected at the operablestage and curative resection is performed. ${ }^{3-5}$ Tumor-related factors and diagnostic stages are considered in RCC and other tumors in predicting disease recurrence, treatment response, or survival. ${ }^{6}$ The course of patients with the same stage and similar histological features is often different suggestive of different dynamics. ${ }^{7}$ So, it is required to search for different markers to predict the prognosis better to determine the risk of mortality and create optimal treatment and follow-up strategies.

Correspondence to: Dr. Ferhat Ekinci, Department of Medical Oncology, Celal Bayar University, Manisa, Turkey E-mail: drferhatekinci@hotmail.com

Received: October 11, 2021; Revised: November 27, 2021; Accepted: December 30, 2021

DOI: https://doi.org/10.29271/jcpsp.2022.03.313
Inflammation mediators are a component of the tumor microenvironment in RCC and can mediate oncogenic differentiation, which may lead to an invasion, angiogenesis, and metastasis in the tumor ${ }^{8,9}$ The mediators that best reflect the increase in these cytokines in peripheral blood are C-reactive protein (CRP), platelet, lymphocyte and neutrophil. ${ }^{8,10}$ Many combinations of these markers in RCC have been studied for many years both in determining recurrence and predicting treatment response and prognosis in the form of neutrophil/lymphocyte ratio (NLR), platelet/lymphocyte ratio (TLR), and Glasgow prognostic score (using CRP and albumin). ${ }^{8,10}$ Another established marker or method such as international metastatic RCC database consortium (IMDC), risk scoring has not yet been agreed on.

Hemoglobin, albumin, lymphocyte, and platelet (HALP) combination, which was first studied in gastric cancer (GC) in 2015, has been reported to be closely related to the clinicopathological features of the disease. It has been studied in many cancers after showing that it is an independent prognostic factor in GC patients. ${ }^{8-11}$ Unlike other combination markers, it was formulated with hemoglobin and albumin, two of the most common indices reflecting patients' performance and nutritional status. ${ }^{10,11}$ It was formulated considering that the decrease in hemoglobin, albumin, and lymphocyte and the increase in platelet were nega- 
tively correlated with the prognosis. ${ }^{11}$ In this sense, since there is only one study conducted in metastatic RCC, this study was planned to contribute to the clinical value of HALP combination.

\section{METHODOLOGY}

The information of patients with metastatic RCC, who were followed up from the Medical Oncology Clinics at the Celal Bayar University, Manisa, Turkey and Adnan Menderes University, Aydin, Turkey, from January 2014 to April 2020 was reviewed retrospectively. Patients who were histologically diagnosed with RCC and at least one metastatic deposit staged based on American Joint Committee on Cancer (AJCC) 8th edition criteria were included. Those patients with sufficient follow-up data were included in the study as a retrospective cohort. Clinico-pathologicalvariablessuch asage, gender, performancestatus(PS), treatments received, histopathology type, metastasis localisation, comorbidities, IMDC risk classification and platelet, lymphocyte, haemoglobin, and serum albumin values that were examined at the time of metastasis were recorded with an electronic medical record system. The PS of the patients was calculated based on the Karnofsky performance status. Initial treatment modalities included surgery, targeted treatment and immunotherapy. Patients who were aged $<18$ years, with non-metastatic RCC, a secondary malignancy and/or did not have adequate laboratory results were excluded from the study.

HALP score was calculated as hemoglobin $(\mathrm{g} / \mathrm{L}) \times$ albumin $(\mathrm{g} / \mathrm{L})$ levels $\times$ lymphocyte count (/L)/platelet count (/L). The endpoints of this study included overall survival (OS) and progression-free survival (PFS). Tumor response was assessed according to response evaluation criteria in solid tumors (RECIST). The cut-off value was determined by calculating the sensitivity and specificity values for the HALP value based on OS and PFS and examining the area under the ROC curve. According to this cut-off value, the patients were divided into Group 1 (low HALP) and Group 2 (high HALP).

The association between the clinicopathological data and the HALP score was evaluated using chi-square test. HALP score and OS-PFS correlations were evaluated using Kaplan-Meier curves with log-rank statistics. Normally distributed variables were analysed by student t-test and other variables were analysed with Mann-Whitney U-test and Kruskal-Wallis test. While examining the normality distribution of quantitative data according to categorical variables, the Shapiro-Wilk test was used in those with $n<30$ group numbers, while the Kolmogorov-Smirnov test was used in those with $n>30$. Furthermore, univariate and multivariate Cox regression analyses were used to calculate the respective hazard ratios (HRs) and 95\% confidence intervals (Cls). Analysis results were presented as median (minimum-maximum). All statistical assessments were two-sided, and a p-value of 0.05 was considered statistically significant.

\section{RESULTS}

A total of $123 \mathrm{mRCC}$ patients were evaluated retrospectively; where $74.8 \%(n=92)$ were males, and $25.2 \%(n=31)$ were females. The median age was 64 (ranging from 21 to 81 ) years. However, $69.9 \%(n=86)$ of the patients were stage 4 at the time of diagnosis. In terms of metastasis localisations, it was noted that the lung was the organ with the highest number of metastatic foci with $69.1 \%(n=85)$. When the pathologies of the patients were examined, it was seen that $79.7 \%(n=98)$ of them had clear cells. Patients were grouped according to IMDC risk classification. The favourable, intermediate, and poor-risk groups had 22 (17.9\%), 69 (56.1\%), and 32 (26\%) patients, respectively. On the follw-up, $52.8 \%(n=65)$ of the patients were dead, and $47.2 \%(n=58)$ of the patients were still alive.

Those calculated as below 0.277 , which is the cut-off value of the HALP score calculated using the ROC curve, were categorised as Group 1, and those calculated as 0.277 and above were categorised as Group 2. There was a significant difference in favour of Group 1 in terms of other metastases $(p=0.001)$. Smokers in Group 2 and non-smokers in group 1 were predominant, but ex-smoker rates were similar $(p=0.033)$. In terms of IMDC risk scoring, it is noteworthy that the favourable risk group is predominant in group 2 and the poor-risk group in Group 1; and the groups are similarly distributed in terms of intermediate risk ( $p=0.002)$. When the ex-status was examined, there was a significant increase in favour of Group 1 ( $p=0.029$, Tablel).

IMDC risk classification, one of the most commonly used and predictive indicators of prognosis in RCC, was similarly significant in predicting PFS and OS in this study. Here, OS was 116.6 months (95\% Cl, 59.70 - 173.49) in the favourable-risk group, 63.9 months $(95 \% \mathrm{Cl}, 37.39$ - 90.40) in the Intermediate-risk group and 15.5 months $(95 \% \mathrm{Cl}, 3.28$ - 27.71) in the poor-risk group $(p<0.001)$. Considering the relationship between IMDC risk scoring and PFS, it was reported as 115.4 months $(95 \% \mathrm{Cl}, 0$ 238.62 ) in the favorable-risk group, 25.1 months ( $95 \% \mathrm{Cl}, 13.33$ $36.93)$ in the Intermediate-risk group, and 11 months $(95 \% \mathrm{Cl}$, 5.38 - 16.61) in the poor-risk group $(p=0.002)$.

The OS of Group 1 patients with a cut-off value lower than 0.277 , which is the determined cut-off value of the HALP score, which constitutes the planning goal of this study, was calculated as 17.7 months $(95 \% \mathrm{Cl}, 2.21$ - 33.18), while the OS of Group 2 patients with a cut-off value higher than 0.277 was calculated as 89.7 months $(95 \% \mathrm{Cl}, 55.62$ - 123.77), and the difference was found to be statistically significant $(p=0.001)$. Again, in Group 2, the risk of death was 0.187 times $(P=0.009)$ compared to the univariate analysis and 0.143 times $(p=0.012)$ less than the multivariate analysis. This result concludes that the HALP score is a robust parameter in predicting survival.

When the patients were categorised according to karnofsky PS, OS of those with PS below $70 \%$ was reported as 10.2 months (95\% $\mathrm{Cl}, 3.27$ - 17.12) and those above $70 \%$ as 69.6 months (52.95 86.24), and the difference was statistically significant ( $p<0,001$ ). When the patients were examined by dividing them into histopathological subgroups, the OS of those with clear cells was 63.9 months (95\% Cl, 41.30 - 86.49), and those with non-clear cells were 9.3 months $(95 \% \mathrm{Cl}, 7.45-11.14)$, and the difference was found to be statistically significant $(p=0.028)$. When examined according to metastasis regions, the OS of those without liver 
metastasis was 71 months ( $95 \% \mathrm{Cl}, 52.29$ - 89.70), while the OS of those with liver metastasis was 9.3 months ( $95 \% \mathrm{Cl}, 3.43-15.16)$ $(p<0.001)$. The PFS of those without liver metastasis was 31.7 months $(95 \% \mathrm{Cl}, 11.66-51.80)$, and of those with liver metastasis was 8.6 months $(95 \% \mathrm{Cl}, 4.84-12.35)(p=0.017)$. It is seen that liver metastasis predicts both OS and PFS statistically significantly. A similar situation is seen in the case of bone metastasis. OS was 71 months (52.29 - 89.70) in those without bone metastasis, while it was 9.3 months (3.43 - 15.16) in those without bone metastasis $(p<0.001)$. Again, in terms of PFS, it was 31.7 months (11.66 - 51.80) in those without bone metastasis and 8.6 months (4.84 - 12.35) in those with bone metastasis $(p=0.017$, Tables II and III). Risk factors affecting overall survival were analyzed by Cox regressionanalysis as univariate and multivariate models.

\section{DISCUSSION}

Many studies have shown that nutritional status and inflammatory response play a central role in cancer disease progression. ${ }^{7-11}$ In general, approximately one-third of cancer patients have cancer-related anemia at the time of diagnosis, which is associated with further cancer stages. ${ }^{8,12-14}$ Albumin level, which acts as both an indicator of nutritional status and a negative acute phase reactant, is also a parameter that has been proven to be associated with prognosis in RCC. ${ }^{15}$ The superiority of hemoglobin and albumin in HALP scoring over other hematological and biochemical parameter combinations is that it also provides clinical information related to nutrition. Decreased lymphocyte count may be responsible for a weak and inadequate immune response to tumors, so it has been associated with poor prognosis, just like neutrophil and platelet increase. ${ }^{8,9}$ Platelets, on the other hand, support the extravasation of the tumor cell by increasing endothelial permeability through vascular endothelial growth factor (VEGF) and giving invasion and metastatic potential to tumor cells. Platelets surprisingly prevent attacks from natural killer (NK) cells by forming a barrier around tumor cells. ${ }^{10}$ Due to direct contact between tumor cells and platelets, epithelial and mesenchymal transition in tumor cells is encouraged, and signalling pathways supporting metastasis are triggered $^{8}$. Another remarkable point in terms of platelet is that the function of granulocytes depends on the presence of platelets, especially in metastatic niche formation. The decrease in platelet level results in a more potent metastasis inhibition than the decrease in granulocyte level alone strengthens this hypothesis. ${ }^{8,9,16}$

Table I: Comparison of categorical variables according to HALP cut-off value.

\begin{tabular}{|c|c|c|c|c|c|}
\hline & Total (n-\%) & $<0.277(n=53)$ & $\geq 0.277(n=70)$ & Total $(n=123)$ & $\mathbf{p}$ \\
\hline \multicolumn{6}{|l|}{ Age category } \\
\hline$<65$ years old & $69(56.1)$ & $25(47.2 \%)$ & $44(62.9 \%)$ & $69(56.1 \%)$ & \multirow{2}{*}{0.083} \\
\hline$\geq 65$ years old & $54(43.9)$ & $28(52.8 \%)$ & $26(37.1 \%)$ & $54(43.9 \%)$ & \\
\hline \multicolumn{6}{|l|}{ Gender } \\
\hline Female & $31(25.2)$ & $16(30.2 \%)$ & $15(21.4 \%)$ & $31(25.2 \%)$ & \multirow{2}{*}{0.268} \\
\hline Male & $92(74.8)$ & $37(69.8 \%)$ & $55(78.6 \%)$ & $92(74.8 \%)$ & \\
\hline \multicolumn{6}{|l|}{ Smoking } \\
\hline Active smoker & $45(36.6)$ & $14(26.4 \%)$ & $31(44.3 \%)$ & $45(36.6 \%)$ & \multirow{3}{*}{0.033} \\
\hline Ex smoker & $31(25.2)$ & $12(22.6 \%)$ & $19(27.1 \%)$ & $31(25.2 \%)$ & \\
\hline Non-smoker & $47(38.2)$ & $27(50.9 \%)$ & $20(28.6 \%)$ & $47(38.2 \%)$ & \\
\hline \multicolumn{6}{|l|}{ Karnofsky PS } \\
\hline$<70 \%$ & $22(17.9)$ & $12(22.6 \%)$ & $10(14.3 \%)$ & $22(17.9 \%)$ & \multirow{2}{*}{0.231} \\
\hline$\geq 70 \%$ & $101(82.1)$ & $41(77.4 \%)$ & $60(85.7 \%)$ & $101(82.1 \%)$ & \\
\hline \multicolumn{6}{|l|}{ Comorbid disease } \\
\hline DM & $20(16.3)$ & $7(13.2 \%)$ & $13(18.6 \%)$ & $20(16.3 \%)$ & 0.425 \\
\hline HT & $44(35.8)$ & $16(30.2 \%)$ & $28(40.0 \%)$ & $44(35.8 \%)$ & 0.261 \\
\hline CRF & $16(13.0)$ & $6(11.3 \%)$ & $10(14.3 \%)$ & $16(13.0 \%)$ & 0.628 \\
\hline CAD & $23(18.7)$ & $11(20.8 \%)$ & $12(17.1 \%)$ & $23(18.7 \%)$ & 0.611 \\
\hline COPD & $9(7.3)$ & $4(7.5 \%)$ & $5(7.1 \%)$ & $9(7.3 \%)$ & 0.932 \\
\hline \multicolumn{6}{|l|}{ Diagnosis stage } \\
\hline Stage 1 & $18(14.6)$ & $5(9.4 \%)$ & $13(18.6 \%)$ & $18(14.6 \%)$ & \multirow{4}{*}{0.203} \\
\hline Stage 2 & $4(3.3)$ & $2(3.8 \%)$ & $2(2.9 \%)$ & $4(3.3 \%)$ & \\
\hline Stage 3 & $15(12.2)$ & $4(7.5 \%)$ & $11(15.7 \%)$ & $15(12.2 \%)$ & \\
\hline Stage 4 & $86(69.9)$ & $42(79.2 \%)$ & $44(62.9 \%)$ & $86(69.9 \%)$ & \\
\hline \multicolumn{6}{|l|}{ Histological type } \\
\hline Clear cell & $98(79.7)$ & $39(73.6 \%)$ & $59(84.3 \%)$ & $98(79.7 \%)$ & \multirow{2}{*}{0.144} \\
\hline Non clear cell & $25(20.3)$ & $14(26.4 \%)$ & $11(15.7 \%)$ & $25(20.3 \%)$ & \\
\hline \multicolumn{6}{|l|}{ Metastasis Status } \\
\hline Liver metastasis & $31(25.2)$ & $17(32.1 \%)$ & $14(20.0 \%)$ & $31(25.2 \%)$ & 0.127 \\
\hline Lung metastasis & $85(69.1)$ & $37(69.8 \%)$ & $48(68.6 \%)$ & $85(69.1 \%)$ & 0.883 \\
\hline Bone metastasis & $40(32.5)$ & $17(32.1 \%)$ & $23(32.9 \%)$ & $40(32.5 \%)$ & 0.927 \\
\hline Brain metastasis & $10(8.1)$ & $5(9.4 \%)$ & $5(7.1 \%)$ & $10(8.1 \%)$ & 0.744 \\
\hline LN metastasis & $78(63.4)$ & $35(66.0 \%)$ & $43(61.4 \%)$ & $78(63.4 \%)$ & 0.599 \\
\hline Other metastasis & $53(43.1)$ & $32(60.4 \%)$ & $21(30.0 \%)$ & $53(43.1 \%)$ & 0.001 \\
\hline \multicolumn{6}{|l|}{ IMDC risk } \\
\hline Favorable risk & $22(17.9)$ & $3(5.7 \%)$ & $19(27.1 \%)$ & $22(17.9 \%)$ & \multirow{3}{*}{0.002} \\
\hline Intermediate risk & $69(56.1)$ & $30(56.6 \%)$ & $39(55.7 \%)$ & $69(56.1 \%)$ & \\
\hline Poor risk & $32(26.0)$ & $20(37.7 \%)$ & $12(17.1 \%)$ & $32(26.0 \%)$ & \\
\hline \multicolumn{6}{|l|}{ Exitus status } \\
\hline No & $58(47.2)$ & $19(35.8 \%)$ & $39(55.7 \%)$ & $58(47.2 \%)$ & \multirow{2}{*}{0.029} \\
\hline Yes & $65(52.8)$ & $34(64.2 \%)$ & $31(44.3 \%)$ & $65(52.8 \%)$ & \\
\hline
\end{tabular}


Table II: Comparison of the OS and PFS times according to the characteristics of the patients.

\begin{tabular}{|c|c|c|c|c|}
\hline & \multicolumn{2}{|c|}{ os } & \multicolumn{2}{|c|}{ PFS } \\
\hline & Median (\%95 Cl Min-Max) & $\mathbf{p}$ & Median (\%95 Cl Min- Max) & $\mathbf{p}$ \\
\hline \multicolumn{5}{|c|}{ Mevian ( } \\
\hline$<65$ years old & $62.7(41.59-83.80)$ & \multirow{2}{*}{0.409} & $25.1(9.66-40.59)$ & \multirow{2}{*}{0.516} \\
\hline$\geq 65$ years old & $56.7(8.87-104.53)$ & & $29.0(3.41-54.52)$ & \\
\hline \multicolumn{5}{|l|}{ Gender } \\
\hline Female & $35.8(8.59-63.00)$ & \multirow{2}{*}{0.220} & $28.7(2.38-54.95)$ & \multirow{2}{*}{0.311} \\
\hline Male & $69.6(34.72-104.47)$ & & $29.0(0-58.78)$ & \\
\hline \multicolumn{5}{|l|}{ Smoking } \\
\hline Active smoker & $62.7(25.05-100.34)$ & \multirow{3}{*}{0.991} & $21.8(0.31-43.28)$ & \multirow{3}{*}{0.889} \\
\hline Ex smoker & $56.7(14.57-98.82)$ & & $25.1(11.05-39.21)$ & \\
\hline Non smoker & $55.2(12.11-98.28)$ & & $30.6(10.07-51.06)$ & \\
\hline \multicolumn{5}{|l|}{ Karnofsky } \\
\hline$<70 \%$ & $10.2(3.27-17.12)$ & \multirow{2}{*}{$<0.001$} & $7.3(0.88-13.64)$ & \multirow{2}{*}{0.103} \\
\hline$\geq 70 \%$ & $69.6(52.95-86.24)$ & & $29.0(17.65-40.27)$ & \\
\hline DM & $89.7(29.94-149.45)$ & 0.708 & $31.7(18.40-45.06)$ & 0.664 \\
\hline HT & $55.2(12.9-97.5)$ & 0.946 & $30.6(17.36-43.77)$ & 0.806 \\
\hline CRF & $95.7(7.92-183.47)$ & 0.202 & $76.2(9.00-143.39)$ & 0.092 \\
\hline CAD & $47.6(7.32-87.87)$ & 0.687 & $26.7(16.76-36.64)$ & 0.728 \\
\hline COPD & $56.7(0-115.60)$ & 0.734 & $12.8(9.14-16.39)$ & 0.453 \\
\hline \multicolumn{5}{|l|}{ Diagnosis stage } \\
\hline Stage 1 & $71(68.49-73.50)$ & \multirow{3}{*}{0.014} & $64.5(43.96-85.09)$ & \multirow{3}{*}{0.018} \\
\hline Stage 3 & $89.7(53.92-125.47)$ & & \begin{tabular}{|l|l}
--- \\
\end{tabular} & \\
\hline Stage 4 & $31.5(8.64-54.35)$ & & $10.9(7.37-14.42)$ & \\
\hline \multicolumn{5}{|l|}{ Histological type } \\
\hline Clear cell & $63.9(41.30-86.49)$ & \multirow{2}{*}{0.028} & $30.6(19.46-41.67)$ & \multirow{2}{*}{0.276} \\
\hline Non clear cell & $9.3(7.45-11.14)$ & & $5.7(0.62-10.77)$ & \\
\hline Liver metastasis & $9.3(3.43-15.16)$ & $<0.001$ & $8.6(4.84-12.35)$ & 0.017 \\
\hline Lung metastasis & $35.8(11.77-59.82)$ & 0.131 & $20.9(2.09-39.76)$ & 0.347 \\
\hline Bone metastasis & $9.3(3.43-15.16)$ & $<0.001$ & $8.6(4.84-12.35)$ & 0.017 \\
\hline Brain metastasis & $84.7(0-183.53)$ & 0.527 & $30.6(0-104.00)$ & 0.266 \\
\hline LN metastasis & $28.7(0-65.16)$ & 0.386 & $20.9(6.19-35.67)$ & 0.352 \\
\hline Other metastasis & $9.3(3.43-15.16)$ & 0.010 & $10.9(4.03-17.76)$ & 0.014 \\
\hline \multicolumn{5}{|l|}{ IMDC risk } \\
\hline Favorable risk & $116.6(59.70-173.49)$ & & $115.4(0-238.62)$ & \\
\hline Intermediate risk & $63.9(37.39-90.40)$ & \multirow{2}{*}{$<0.001$} & $25,1(13.33-36.93)$ & \multirow{2}{*}{0.002} \\
\hline Poor risk & $15.5(3.28-27.71)$ & & $11(5.38-16.61)$ & \\
\hline \multicolumn{5}{|l|}{ HALP } \\
\hline$<0.277$ & $17.7(2.21-33.18)$ & \multirow{2}{*}{0.001} & $11(0-33.34)$ & \multirow{2}{*}{0.110} \\
\hline$\geq 0.277$ & & & $31.5(7.50-55.43)$ & \\
\hline
\end{tabular}

Table III: COX regression analysis results of the OS.

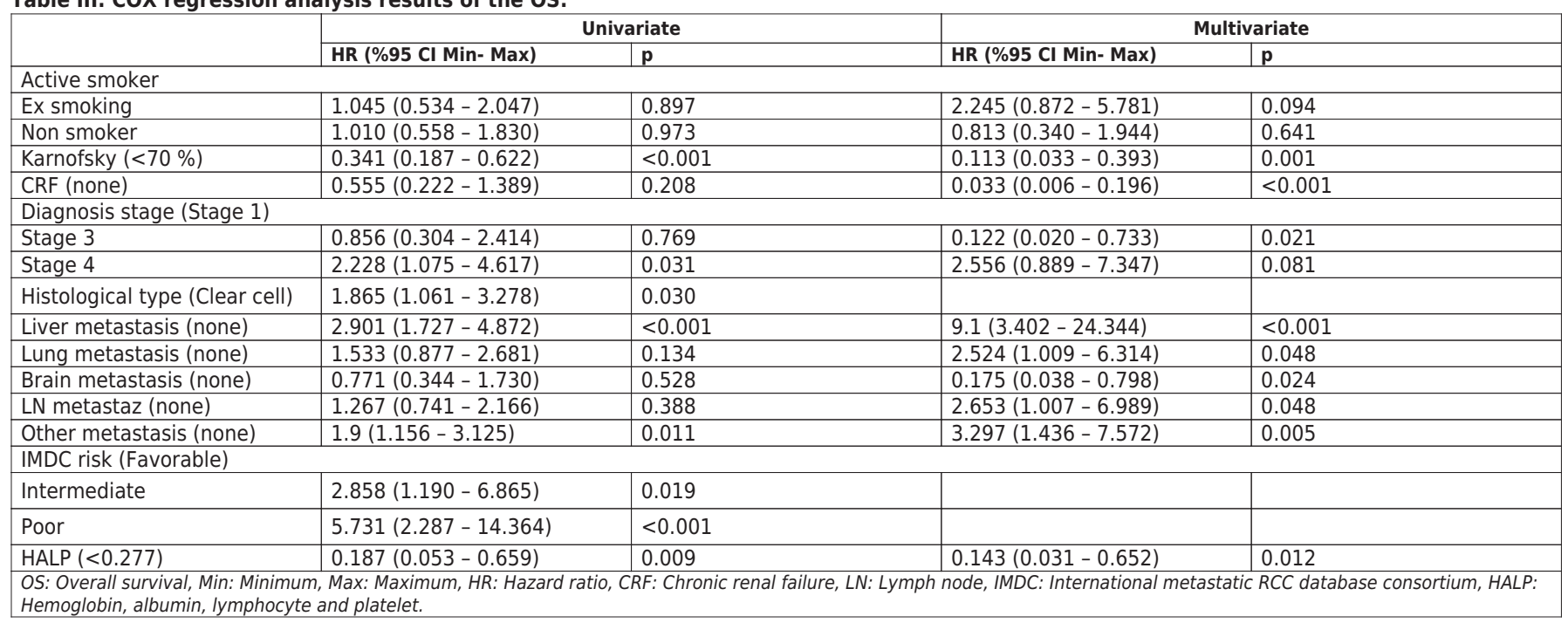

In the light of all these data, the HALP scoring created by formulating hemoglobin, albumin, lymphocyte, and platelet values of patients diagnosed with gastric cancer in 2015 for the first time before the operation is an independent prog- 
nostic factor in the disease and closely related to clinicopathological features. ${ }^{8-11}$ Among the values used in the formulation, it was concluded that the increase in albumin, lymphocyte, and hemoglobin levels was associated with a good prognosis. Still, the increase in platelet level was associated with a poor prognosis, as expected. It has been shown that patients with high HALP scores have a better prognosis than those with low HALP scores. ${ }^{11,12}$ Immediately after this positive result, those with a low HALP score in the scoring calculated with the parameters examined in the preoperative period in the patient population diagnosed with locally advanced colorectal cancer were associated with a higher risk of death (HR: $3.29(95 \% \mathrm{Cl}$ 2.48-4.36; $p$ $<0.001$ ). Following these studies, a similar positive relationship has been proven in many cancer types such as pancreas, esophagus, bladder, and small cell lung cancer. ${ }^{11,12,17-20}$

The first study demonstrating the prognostic significance of the HALP score in RCC was conducted by Peng et al. on 1360 patients who underwent nephrectomy by the urology clinic in $2018 .^{7}$ In that study, unlike this study, preoperative values were taken into consideration according to the HALP scoring, which was presently calculated based on the values examined during metastasis. While clear cell histology was dominant in both studies, a significant difference of $79.7 \%$ was noted in our study and $90.2 \%$ in the study by Peng et al. Another important detail was that the patients with stage 4 at the time of diagnosis were $69.9 \%$ in this study. In comparison, it was only $0.5 \%$ in the study of Peng et al. As a final result of this study, Kaplan-Meier and log-rank tests showed that HALP was strongly associated with cancer-specific survival $(p<0.001)$ and was an independent prognostic factor in multivariate analysis $(p=0.002)$. This relationship has been proven for the first time in RCC, as in many other types of cancer mentioned earlier. In our study, the relationship between carnofsky PS $(p=0.001)$, CRF disease history $(p<0.001)$, stage 3 disease at the time of diagnosis $(p=0.021)$, liver metastasis $(p<0.001)$, lung metastasis $(p=0.048)$, brain metastasis $(p=0.024)$, lymph node metastasis $(p=0.048)$, other metastasis $(p=0.005)$ and HALP score $(p=0.012)$ were shown to be strong and independent according to the results of the multivariate OS analysis.

A very recent study has recently investigated the predictive importance of the HALP score in patients with metastatic RCC treated with nivolumab, a programmed death-1 (PD-1) receptor inhibitor. ${ }^{21}$ The data of 45 patients who used nivolumab were analysed retrospectively. The PFS calculated in the group with a high HALP score was statistically significantly longer than the low group (12.0 months vs 6.0 months $p<0.05)$. The relationship between the HALP score and immunotherapy response has not yet been examined in the literature can be considered the most valuable aspect of this study.
The major limitation of this study is that a retrospective analysis method with potential bias was used in patient selection. Another point is that the HALP score does not have an ideal cut-off value that prevents routine use.

\section{CONCLUSION}

HALP score examined in metastatic RCC patients was closely related to the prognosis. Worse OS has been reported in patients with a low HALP score. Studies involving more patients and requiring prospective design are needed to confirm these results.

\section{ETHICAL APPROVAL:}

The Health Sciences Ethics Committee approved this study of Manisa Celal Bayar University Faculty of Medicine with the decision dated 27/11/2019 and No. 20.478.486.

\section{PATIENTS' CONSENT:}

Written consents were obtained from all the participants.

\section{CONFLICT OF INTEREST:}

The authors declared no conflict of interest.

\section{AUTHORS' CONTRIBUTION:}

FE: Concept, supervision, materials, analysis, interpretation, literature search and writing manuscript.

OYB: Data collection, processing, analysis and interpretation. EO: Data collection, processing and critical review.

APE: Supervision, literature search and critical review.

\section{REFERENCES}

1. Capitanio U, Bensalah K, Bex A, Boorjian SA, Bray F, Coleman J, et al. Epidemiology of renal cell carcinoma. Eur Urol 2019; 75(1):74-84. doi: 10.1016/j.eururo.2018.08.036.

2. Siegel RL, Fedewa SA, Miller KD, Goding-Sauer A, Pinheiro PS, Martinez-Tyson D, et al. Cancer statistics for hispanics/latinos, 2015. CA Cancer J Clin 2015; 65(6):457-80. doi.10.3322/caac. 21314 .

3. Capitanio U, Montorsi F. Renal cancer. Lancet 2016; 387(10021):894-906. doi:10.1016/S0140-6736(15)00046-X.

4. Battaglia M, Lucarelli $G$. The role of renal surgery in the era of targeted therapy: The urologist's perspective. Urologia 2015; 82(3):137-8. doi:10.5301/uro.5000105.

5. Lee-Ying R, Lester R, Heng DY. Current management and future perspectives of metastatic renal cell carcinoma. Int J Urol 2014; 21(9):847-55. doi:10.111/iju.12502.

6. Ekinci F, Erdoğan AP, Dirican A, Göksel G. The effect of neutrophil lymphocyte ratio on prognosis in patients with metastatic renal cell carcinoma. Abant Med J 2021; 10(1):134-9. doi: 10.47493/abantmedj.2021.43.

7. Peng D, Zhang CJ, Tang Q, Zhang L, Yang KW, Yu XT, et al. Prognostic significance of the combination of preoperative hemoglobin and albumin levels and lymphocyte and platelet counts (HALP) in patients with renal cell carcinoma after nephrectomy. BMC Urol 2018; 18(1):20. doi:10.1186/ s12894-018-0333-8. 
8. Greten FR, Grivennikov SI. Inflammation and cancer: Triggers, mechanisms, and consequences. Immunity 2019; 51(1):27-41. doi: 10.1016/j.immuni.2019.06.025.

9. Dirican A, Ekinci $F$, Erdoğan AP, Göksel G. Inflammatory prognostic index score as a new parameter predicting overall survival in renal cell carcinoma. J Surgery Med 2021; 5(2):163-7. doi: 10.28982/josam.850739.

10. Singh R, Mishra MK, Aggarwal H. Inflammation, Immunity, and cancer. Mediators Inflamm 2017; 2017:6027305. doi: 10.1155/2017/6027305.

11. Chen XL, Xue L, Wang W, Chen HN, Zhang WH, Liu K, et al. Prognostic significance of the combination of preoperative hemoglobin, albumin, lymphocyte and platelet in patients with gastric carcinoma: A retrospective cohort study. Oncotarget 2015; 6(38):41370-82. doi: 10.18632/oncotarget.5629.

12. Guo Y, Shi D, Zhang J, Mao S, Wang L, Zhang W, et al. The hemoglobin, albumin, lymphocyte, and platelet (HALP) score is a novel significant prognostic factor for patients with metastatic prostate cancer undergoing cytoreductive radical prostatectomy. J Cancer 2019; 10(1):81-91 doi: 10.7150/jca.27210. doi: 10.7150/jca.27210.

13. Peng D, Zhang CJ, Gong YQ, Hao H, Guan B, Li XS, et al. Prognostic significance of HALP (hemoglobin, albumin, lymphocyte and platelet) in patients with bladder cancer after radical cystectomy. Sci Rep 2018; 8(1):794. doi: 10.1038/s41598-018-19146-y.

14. Komura K, Hashimoto T, Tsujino T, Muraoka R, Tsutsumi T, Satake $\mathrm{N}$, et al. The CANLPH score, an integrative model of systemic inflammation and nutrition status (SINS), predicts clinical outcomes after surgery in renal cell carcinoma: Data from a multicenter cohort in japan. Ann Surg Oncol 2019;
26(9):2994-3004. doi:10.1245/s10434-019-07530-5.

15. Stenman M, Laurell A, Lindskog M. Prognostic significance of serum albumin in patients with metastatic renal cell carcinoma. Med Oncol 2014; 31(3):841. doi: 10.1007/s12032014-0841-7.

16. Labelle M, Begum S, Hynes RO. Platelets guide the formation of early metastatic niches. Proc Natl Acad Sci U S A 2014; 111(30):E3053-61. doi: 10.1073/pnas.1411082111.

17. Jiang $H$, Li H, Li A, Tang E, Xu D, Chen Y, et al. Preoperative combined hemoglobin, albumin, lymphocyte and platelet levels predict survival in patients with locally advanced colorectal cancer. Oncotarget 2016; 7(44):72076-83. doi:10.18632/oncotarget.12271.

18. Xu SS, Li S, Xu HX, Li H, Wu CT, Wang WQ, et al. Haemoglobin, albumin, lymphocyte and platelet predicts postoperative survival in pancreatic cancer. World J Gastroenterol 2020; 26(8):828-38. doi: 10.3748/wjg.v26.i8.828.ww

19. Topal U, Dal F, Sozuer EM, Akyuz M, Talih T, Islam DG, et al. Combination of preoperative haemoglobin and albumin levels and lymphocyte and platelet counts (HALP) in patients with oesophageal cancer. J Evolution Med Dent Sci 2021; 10(4):173-8. doi: 10.14260/jemds/2021/38.

20. Yang N, Han X, Yu J, Shu W, Qiu F, Han J. Hemoglobin, albumin, lymphocyte, and platelet score and neutrophil-to-lymphocyte ratio are novel significant prognostic factors for patients with small-cell lung cancer undergoing chemotherapy. J Cancer Res Ther 2020; 16(5):1134-9. doi: 10.4103/jcrtJCRT_1066_19.

21. Yilmaz M. Is there predictive significance of HALP score in metastatic RCC patients treated with nivolumab? EJMI 2021; 5(1):33-8 doi: 10.14744/ejmi.2021.15579. 